\title{
Characteristics of Heat-Annealed Silicon Homojunction Infrared Photodetector Fabricated by Plasma-Assisted Technique
}

\author{
Oday A. HAMMADI* \\ Department of Physics, College of Education, Al-Iraqia University, Baghdad, IRAQ \\ ${ }^{*}$ Corresponding author: Oday A. HAMMADI_ Email: odayata2001@yahoo.com
}

\begin{abstract}
In this work, the effect of thermal annealing on the characteristics of silicon homojunction photodetector was studied. This homojunction photodetector was fabricated by means of plasma-induced etching of p-type silicon substrate and plasma sputtering of n-type silicon target in vacuum. The electrical and spectral characteristics of this photodetector were determined and optimized before and after the annealing process. The maximum surface reflectance of $1.89 \%$ and $1.81 \%$, the maximum responsivity of $0.495 \mathrm{~A} / \mathrm{W}$ and $0.55 \mathrm{~A} / \mathrm{W}$, the ideality factor of 1.80 and 1.99 , the maximum external quantum efficiency of $76 \%$ and $83.5 \%$, and the built-in potential of $0.79 \mathrm{~V}$ and $0.72 \mathrm{~V}$ were obtained before and after annealing, respectively.
\end{abstract}

Keywords: Photodetectors; homojunction; spectral responsivity; plasma-induced etching

Citation: Oday A. HAMMADI, "Characteristics of Heat-Annealed Silicon Homojunction Infrared Photodetector Fabricated by Plasma-Assisted Technique," Photonic Sensors, 2016, 6(4): 345-350.

\section{Introduction}

Homojunction is essentially a junction between the $\mathrm{n}$ - and p-type portions of the same material formed by different impurity doping. The junction is termed as abrupt or graded, depending upon whether the impurity concentration in the material changes abruptly or gradually at the junction region $[1,2]$. When two layers of semiconductor materials of opposite carrier types are intimately joined, an exchange of charge carriers takes place, and the Fermi level becomes continuous in both layers [3]. Electrons from n-type portion adjacent to the junction flow into the p-side while holes from p-type to $\mathrm{n}$ side, and this flow is due to the density gradient. Then there will be some uncompensated stationary charges forming a dipole array, leading to the barrier formation. This equalizes the Fermi levels and prevents further flow of charges to either side $[4,5]$. The energy band diagram of $p-n$ homojunction is shown in Fig. 1.

Silicon photodiodes are responsive to high-energy particles and photons and operated by the absorption of photons or charged particles, generating a flow of current in an external circuit, which is proportional to the incident power. These devices can be used to detect the presence or absence of minute quantities of light with intensities in the range of $10^{-9} \mathrm{~mW} / \mathrm{cm}^{2}-10^{2} \mathrm{~mW} / \mathrm{cm}^{2}$ [6]. Silicon photodiodes are efficiently used in many applications, such as spectroscopy, photography, analytical instrumentation, optical position sensors, beam alignment, surface characterization, laser range finders, optical communications, and medical

Received: 2 May 2016 / Revised: 27 August 2016

(C) The Author(s) 2016. This article is published with open access at Springerlink.com

DOI: $10.1007 / \mathrm{s} 13320-016-0338-4$

Article type: Regular 
imaging instruments $[7,8]$.

The photonic and optoelectronic devices based on silicon diodes still have their featured positions in their applications due to several reasons, among which the natural availability, ease fabrication, and open technology are noticeably observed [9]. Despite the reasonably higher efficiencies achieved by similar devices (e.g., solar cells and photodetectors) fabricated from other semiconducting materials, the lower values of silicon devices can be compensated by their lower costs and higher reproducibility in correlation to the revolution in porous and nanocrystalline silicon structures $[10,11]$.

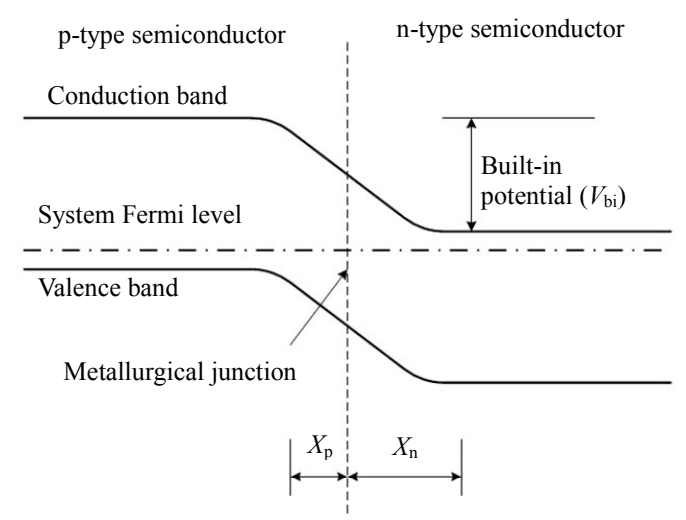

Fig. 1 Energy band diagram of p-n homojunction.

Compared with the avalanche photodiodes, the silicon junction photodetectors have no internal gain but they are very fast $(\sim 0.4 \mathrm{~ns})$ and have very large bandwidths (up to $2.5 \mathrm{GHz}$ ) [12]. As well, silicon homojunctions are the fundamental structures of bipolar and metal-oxide-semiconductor (MOS) transistors, which represent the foundation of modern semiconductor electronics [13].

In the modern semiconducting materials technology, plasma processing techniques present very good candidates and alternatives for the conventional and expensive ones [14, 15]. Construction and operation of plasma systems for semiconductor processing applications can be performed and optimized to a reasonable degree. Such systems can be easily used for low cost and mass production of diverse structures and devices with very good quality $[16,17]$.

In this work, a silicon homojunction was fabricated by plasma-assisted technique and characterized for photodetection applications. Also, the effect of thermal annealing on the characteristics of this homojunction was studied.

\section{Experiment}

Figure 2 shows the experimental steps followed in this work to fabricate the silicon homojunction. A boron-doped p-type silicon wafer with the orientation of $<100>$, electrical resistivity of $40 \mathrm{~cm}-$ $50 \mathrm{~cm}$, diameter of $5 \mathrm{~cm}$, and thickness of $675 \mathrm{~m}$ was used for the p-type side of the proposed homojunction. This wafer was etched by argon discharge plasma for $20 \mathrm{~min}$ and then heat treated for one hour at $150{ }^{\circ} \mathrm{C}$ to get homogeneous rough layer of about $20-\mathrm{nm}$ thickness. The sample was then placed inside the sputtering chamber to deposit an n-type silicon layer on its surface. This chamber was evacuated down to 0.001 mbar to prevent any active contaminants may reside inside from the interaction with the silicon samples.

(a)

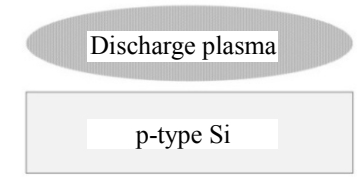

(b)

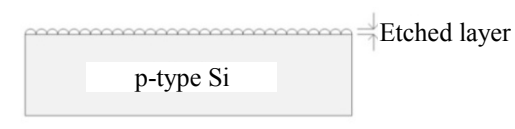

Sputtered n-type Si

(c)

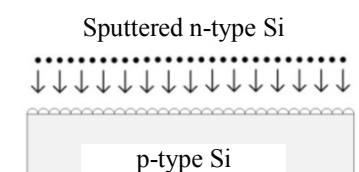

(d)

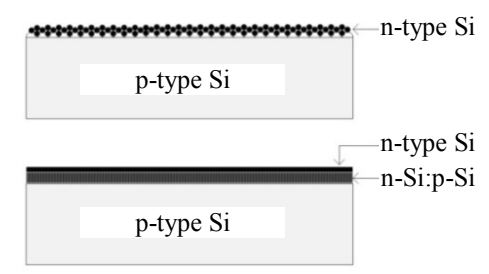

Fig. 2 Fabrication stages of the $\mathrm{Si}$ homojunction: (a) plasma-induced etching, (b) formation of etched Si layer, (c) sputtering of n-type $\mathrm{Si}$ target, (d) deposition of n-type $\mathrm{Si}$ layer, and (e) thermal annealing to form $\mathrm{n}-\mathrm{Si} / \mathrm{p}$-Si homojunction. 
The sputtering target was a phosphorous-doped n-type silicon wafer with the orientation of $<111>$, electrical resistivity of $25 \mathrm{~cm}-30 \mathrm{~cm}$, diameter of $5 \mathrm{~cm}$, and thickness of $675 \mathrm{~m}$. The sputtering target was placed on the cathode while the p-type silicon substrate was placed on the anode and the inter-electrode distance was $4 \mathrm{~cm}$, which was found to be the optimum [18]. The sputtering plasma was generated by the electrical discharge of argon at the pressure of $0.08 \mathrm{mbar}$, discharge voltage of $3.5 \mathrm{kV}$, and discharge current of $45 \mathrm{~mA}$. The sputtering time was 2 hours to form a 30 -nm-thickness n-type silicon layer over the previously formed 20-nm p-type silicon layer. The final sample formed on the p-type substrate was about 30-nm-thickness n-Si/p-Si layer. In order to introduce the effect of thermal annealing on the characteristics of the fabricated structure, the final sample was gradually annealed for one hour at $20{ }^{\circ} \mathrm{C}-320{ }^{\circ} \mathrm{C}$ with a step of $5{ }^{\circ} \mathrm{C} / \mathrm{min}$ to induce the formation of the homogeneous structure. Figure 3 shows the scanning electron microscopy (SEM) micrograph of the formed junction.

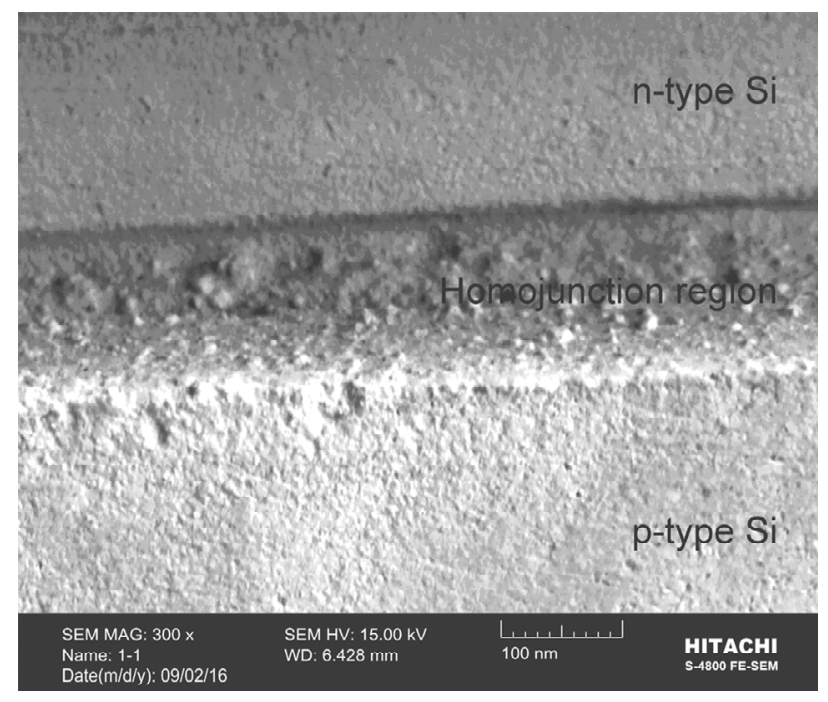

Fig. 3 SEM micrograph of the junction region formed in this work.

The electrical characterization of the fabricated homojunction includes the current-voltage and capacitance-voltage characteristics before and after the thermal annealing process and determination of some parameters, such as ideality factor $(n)$ and built-in potential $\left(V_{\mathrm{bi}}\right)$. The electrical measurements were carried out using a dc power supply $0 \mathrm{~V}-30 \mathrm{~V}$, a Keithley 616 picoammeter, and a Keithley $82 \mathrm{C}-\mathrm{V}$ system.

The optical characterization of the fabricated homojunction includes the measurements of the reflectance from the surface, spectral responsivity, and external quantum efficiency before and after the thermal annealing process. The reflectance was measured by the specular hemispherical method, and the spectral responsivity was measured by using the pulsed xenon lamp, monochromator, bandpass filer, charge-coupled device (CCD) array detector, and data acquisition system. All optical measurements were carried out in the spectral range of $350 \mathrm{~nm}-$ $1100 \mathrm{~nm}$.

\section{Results and discussion}

Figure 4 shows the current-voltage $(I-V)$ characteristics of the prepared silicon homojunction before and after the thermal annealing process. It is clearly observed that these characteristics were slightly enhanced by thermal annealing as they were in accordance to the typical behavior of homojunction. As well, the ideality factor of the fabricated homojunction increased from 1.80 to 1.99 after annealing. This enhancement can be attributed to the reduction in the saturation current, which is in turn related to the reduction in the charge carrier recombination. Thermal annealing of the fabricated device presents better properties for charge carrier separation as a consequence of the application of a potential bias. Therefore, the adequate layer characteristics for charge carrier transfer lead to a reduction in charge carrier recombination making up for the lack of charge carrier separation when applying an electric potential bias.

Figure 5 shows the variation of inverse squared capacitance with the applied voltage $\left(C^{-2}-V\right)$ for the fabricated homojunction before and after thermal annealing. The capacitance of the depletion layer of this homojunction increased due to thermal 
annealing. This increase in the capacitance could be attributed to a decrease in the built-in potential from 0.79 to 0.72 as the width of the depletion layer decreased because of the increasing recombination rate on both sides of this layer.

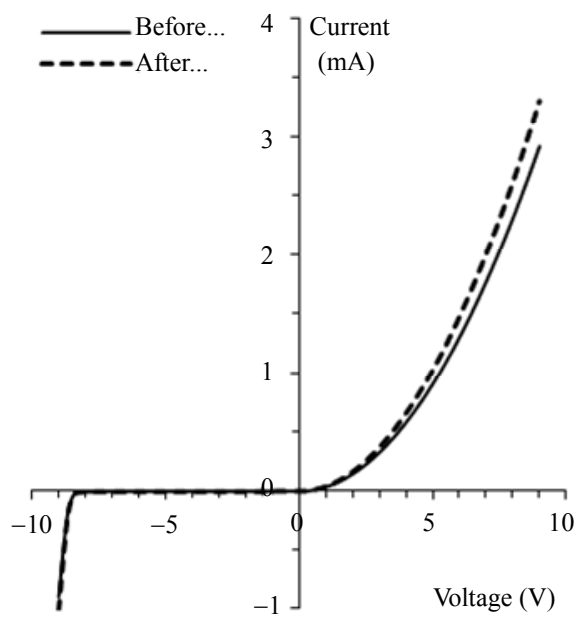

Fig. $4 I-V$ characteristics of the fabricated homojunction before and after thermal annealing.

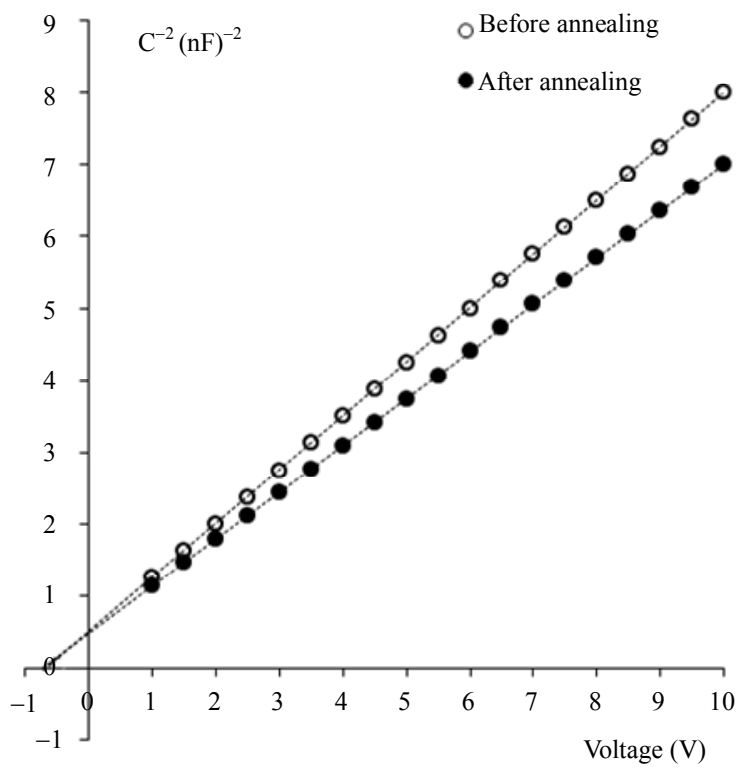

Fig. $5 C^{-2}-V$ characteristics of the fabricated homojunction before and after thermal annealing (the built-in potential is determined to be $0.72 \mathrm{~V}$ before annealing and $0.79 \mathrm{~V}$ after annealing).

Figure 6 shows the effect of thermal annealing on the surface reflectance of the fabricated homojunction as it was reduced from $1.89 \%$ to $1.81 \%$ in the spectral range of $350 \mathrm{~nm}-1100 \mathrm{~nm}$. Accordingly, the absorbance of this homojunction is supposed to increase by $0.08 \%$ due to this reduction in the surface reflectance, and this enhancement in the absorption may lead to the corresponding enhancement in the spectral response of the homojunction.

The spectral responsivity $(R)$ of the fabricated homojunction was measured in the range of $350 \mathrm{~nm}$ - $1100 \mathrm{~nm}$ and presented in Fig. 7. The thermal annealing caused an enhancement in the spectral responsivity from $0.495 \mathrm{~A} / \mathrm{W}$ to $0.55 \mathrm{~A} / \mathrm{W}$ as the annealing process made the homogeneity of the sensitive layer ( $\mathrm{n}-\mathrm{Si} / \mathrm{p}-\mathrm{Si}$ ) structure better to respond to the incident radiation.

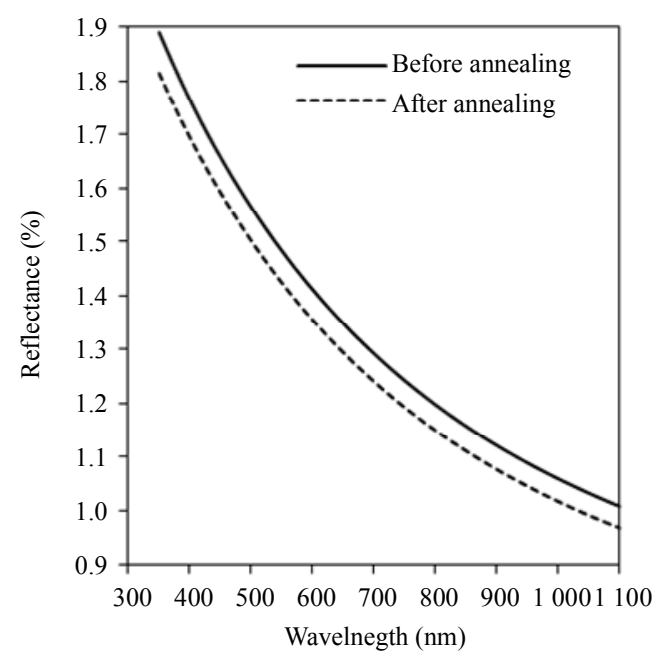

Fig. 6 Surface reflectance of the fabricated homojunction before and after thermal annealing.

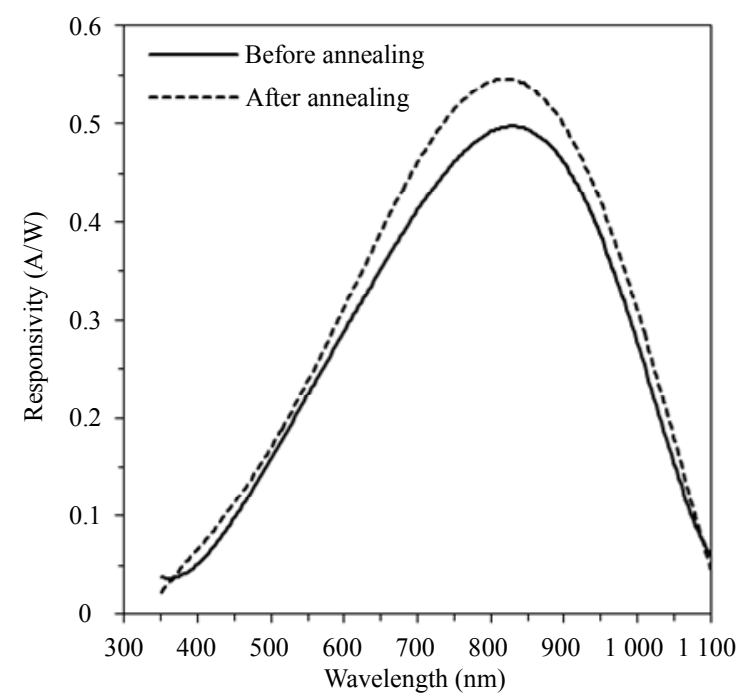

Fig. 7 Spectral responsivity of the fabricated homojunction before and after thermal annealing.

As the fabricated homojunction can be employed 
in many applications of photonics and optoelectronics, the performance of this device should be assessed by introducing its efficiency. Therefore, the external quantum efficiency (EQE) of the fabricated homojunction was determined as a function of the wavelength of incident radiation before and after thermal annealing and was enhanced from $76 \%$ to $83.5 \%$, respectively, as shown in Fig. 8.

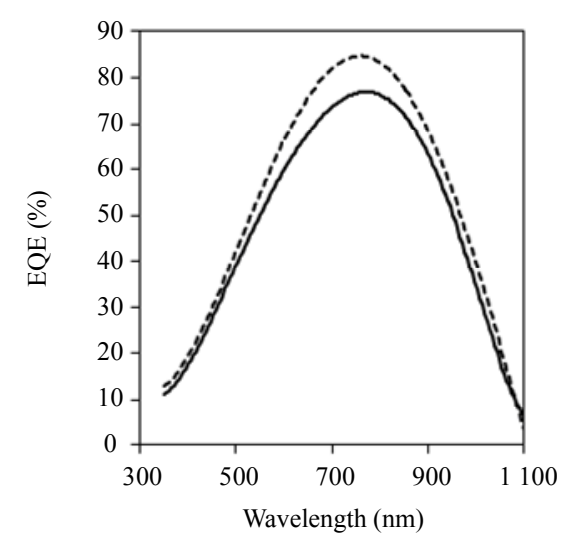

Fig. 8 Quantum efficiency of the fabricated homojucntion photodetector before and after thermal annealing.

\section{Conclusions}

According to the results obtained from this work, the thermal annealing has reasonable effects on the characteristics of silicon homojunction photodetector fabricated by means of the plasmainduced etching of the p-type silicon substrate and plasma sputtering of the n-type silicon target in vacuum. Due to thermal annealing, the maximum surface reflectance and built-in potential of this photodetector noticeably decreased while the maximum responsivity, the ideality factor, and the maximum external quantum efficiency increased. The fabrication procedure used in this work is reasonably new, low-cost, easily controlled, and reliable.

Open Access This article is distributed under the terms of the Creative Commons Attribution 4.0 International License (http://creativecommons.org/ licenses/by/4.0/), which permits unrestricted use, distribution, and reproduction in any medium, provided you give appropriate credit to the original author(s) and the source, provide a link to the Creative Commons license, and indicate if changes were made.

\section{References}

[1] R. A. Ismail, O. A. Abdulrazaq, A. A. Hadi, and O. A. Hamadi, "Full characterization at $904 \mathrm{~nm}$ of large area Si p-n junction photodetectors produced by laser-induced diffusion," International Journal of Modern Physics, 2007, 19(31): 197-201.

[2] M. Consales, M. Pisco, and A. Cusano, "Lab-on-fiber technology: a new avenue for optical nanosensors," Photonic Sensors, 2012, 2(4): 289-314.

[3] C. Chen, H. Wang, Z. Jiang, X. Jin, and J. Luo, "Design, fabrication, and measurement of two silicon-based ultraviolet and blue-extended photodiodes," Photonic Sensors, 2014, 4(4): 373-378.

[4] D. Viveiros, J. Ferreira, S. O. Silva, J. Ribeiro, D. Flores, J. L. Santos, et al., "Ammonia sensing system based on wavelength modulation spectroscopy," Photonic Sensors, 2015, 5(2): 109-115.

[5] J. Dresner and F. V. Shallcross, "Crystallinity and electronic properties of evaporated CdS films," Journal of Applied Physics, 1963, 34(8): 2390-2395.

[6] O. A. Hamadi and K. Z. Yahiya, "Optical and electrical properties of selenium-antimony heterojunction formed on silicon substrate," Journal of Pure and Applied Sciences, 2007, 4(2): 1-11.

[7] O. A. Hamadi, B. A. M. Bader, and A. K. Yousif, "Electrical characteristics of silicon p-n junction solar cells produced by plasma-assisted matrix etching technique," Engineering and Technics, 2008, 26(8): 995-1001.

[8] R. A. Ismail, O. A. Abdulrazaq, A. A. Hadi, and O. A. Hamadi, "Full characterization at $904 \mathrm{~nm}$ of Si p-n junction photodetectors produced by LID technique," International Journal of Modern Physics B, 2007, 19(31): 197-201.

[9] A. K. Yousif and O. A. Hamadi, "Plasma-induced etching of silicon surfaces," Bulgarian Journal Physics, 2008, 35(3): 191-197.

[10] O. A. Hamadi, "Characteristics of CdO-Si heterostructure produced by plasma-induced bonding technique," Proceedings of the Institution of Mechanical Engineers Part L Journal of Materials Design and Applications, 2008, 222: 65-71.

[11] O. A. Hamadi, "Effect of annealing on the electrical characteristics of $\mathrm{CdO}-\mathrm{Si}$ heterostructure produced by plasma-induced bonding technique," Iraqi Journal of Applied Physics, 2008, 4(3): 34-37.

[12] A. A. K. Hadi and O. A. Hamadi, "Optoelectronic characteristics of As-doped silicon photodetectors produced by LID technique," Iraqi Journal of Applied Physics, 2008, 1(2): 23-26. 
[13] O. A. Hammadi, "Photovoltaic properties of thermally-grown selenium-doped silicon photodiodes for infrared detection applications," Photonic Sensors, 2015, 5(2): 152-158.

[14] O. A. Hammadi, M. K. Khalaf, and F. J. Kadhim, "Silicon nitride nanostructures prepared by reactive sputtering using closed-field unbalanced dual magnetrons," Proceedings of the Institution of Mechanical Engineers Part L Journal of Materials Design and Applications, 2015, 229(5).

[15] O. A. Hammadi, M. K. Khalaf, and F. J. Kadhim, "Fabrication of UV photodetector from nickel oxide nanoparticles deposited on silicon substrate by closed-field unbalanced dual magnetron sputtering techniques," Optical and Quantum Electronics, 2015, 47(2): 1-9.
[16] O. A. Hammadi, M. K. Khalaf, and F. J. Kadhim, "Fabrication and characterization of UV photodetectors based on silicon nitride nanostructures prepared by magnetron sputtering," Proceedings of the Institution of Mechanical Engineers Part $N$ Journal of Nanoengineering and Nanosystems, 2015, 230(1): 32-36.

[17] O. A. Hammadi and N. E. Naji, "Electrical and spectral characterization of $\mathrm{CdS} / \mathrm{Si}$ heterojunction prepared by plasma-induced bonding," Optical and Quantum Electronics, 2016, 48(8): 1-7.

[18] O. A. Hammadi, M. K. Khalaf, F. J. Kadhim, and B. T. Chiad, "Operation characteristics of a closed-field unbalanced dual-magnetrons plasma sputtering system," Bulgarian Journal Physics, 2014, 41(1): 24-33. 